\title{
Fourier transforms of UD integrals
}

\author{
Igor Kondrashuk ${ }^{a}$ and Anatoly Kotikov ${ }^{b}$ \\ (a) Departamento de Ciencias Básicas, \\ Universidad del Bío-Bí, Campus Fernando May, Casilla 447, Chillán, Chile \\ (b) Bogoliubov Laboratory of Theoretical Physics, Joint Institute for Nuclear Research, \\ Dubna, Russia
}

\begin{abstract}
UD integrals published by N. Usyukina and A. Davydychev in 1992-1993 are integrals corresponding to ladder-type Feynman diagrams. The results are UD functions $\Phi^{(L)}$, where $L$ is the number of loops. They play an important role in $\mathcal{N}=4$ supersymmetic Yang-Mills theory. The integrals were defined and calculated in the momentum space. In this paper the position space representation of UD functions is investigated. We show that Fourier transforms of UD functions are UD functions of space-time intervals but this correspondence is indirect. For example, the Fourier transform of the second UD integral is the second UD integral.
\end{abstract}

Keywords: UD integrals, UD functions. 


\section{Introduction}

As has been shown in Refs. [1] - [12], Slavnov-Taylor identity predicts that the correlators of dressed mean fields for $\mathcal{N}=4$ supersymmetric Yang-Mills theory in the position space can be represented in terms of UD integrals. The UD integrals correspond to the momentum representation of ladder diagrams and were calculated in Refs. $[15,16]$ in the momentum space, and the result can be written in terms of certain functions (UD functions) of conformally invariant ratios of momenta. Indeed, the $L c c$ correlator in the position space in Wess-Zumino-Landau gauge of maximally supersymmetric Yang-Mills theory is a function of Davydychev integral $J(1,1,1)$ at two loop level ${ }^{1}[5,6,7]$. By using Slavnov-Taylor identity one can represent correlators of dressed mean gluons in terms of this integral at two-loop level. What kind of integrals will contribute to the scale-independent $L c c$ correlator at all loops is unclear at present. However, using a method of Ref. [7] one can suggest that at higher loop orders in the position space the UD integrals will survive only. Conformal invariance of the effective action of dressed mean fields in the position space, predicted in Refs. [1, 2, 3,4] corresponds to the property of conformal invariance of UD integrals.

In the momentum space it was shown that UD functions are the only contributions (at least up to three loops) to off-shell four-point correlator of gluons that corresponds to four gluon amplitude $[17,18]$. The conformal invariance of UD functions was used in the momentum space to calculate four point amplitude and to classify all possible contributions to it [19, 20]. Later, the conformal symmetry in the momentum space appeared on the string side in the AldayMaldacena approach [21] in the limit of strong coupling.

The purpose of this paper is to find the position space representation of the ladder diagrams that produce UD functions in the momentum space. In this paper we show that Fourier transform of the second UD integral is the second UD integral and that Fourier transform of the first UD function can be related to the second UD function. We consider three-point ladder UD integrals and comment four-point ladder UD integrals. In Section 2 we illustrate the idea of the method on an example of the simplest diagram. The most important point is that the problem is solved diagrammatically via conformal transformation. Two other solutions to this problem are given in Section 3 and Section 4.

\section{First UD triangle diagram}

First UD triangle diagram is depicted on the 1.h.s. of Fig. (1). All the notation used in this paper is the notation of Ref. [5]. To calculate it we use conformal transformation. The conformal transformation for each vector of the integrand (including the external vectors) is

$$
y_{\mu}=\frac{y_{\mu}^{\prime}}{y^{\prime 2}}, \quad z_{\mu}=\frac{z_{\mu}^{\prime}}{z^{\prime 2}}
$$

On the level of equations, the chain of conformal transformations of the 1.h.s. of Fig.(1)

\footnotetext{
${ }^{1}$ In the position space Feynman diagrams contain integrations over coordinates of internal vertices. Integration over internal vertices appears in dual representation of the momentum diagrams too (see below and [13, 14]).
} 

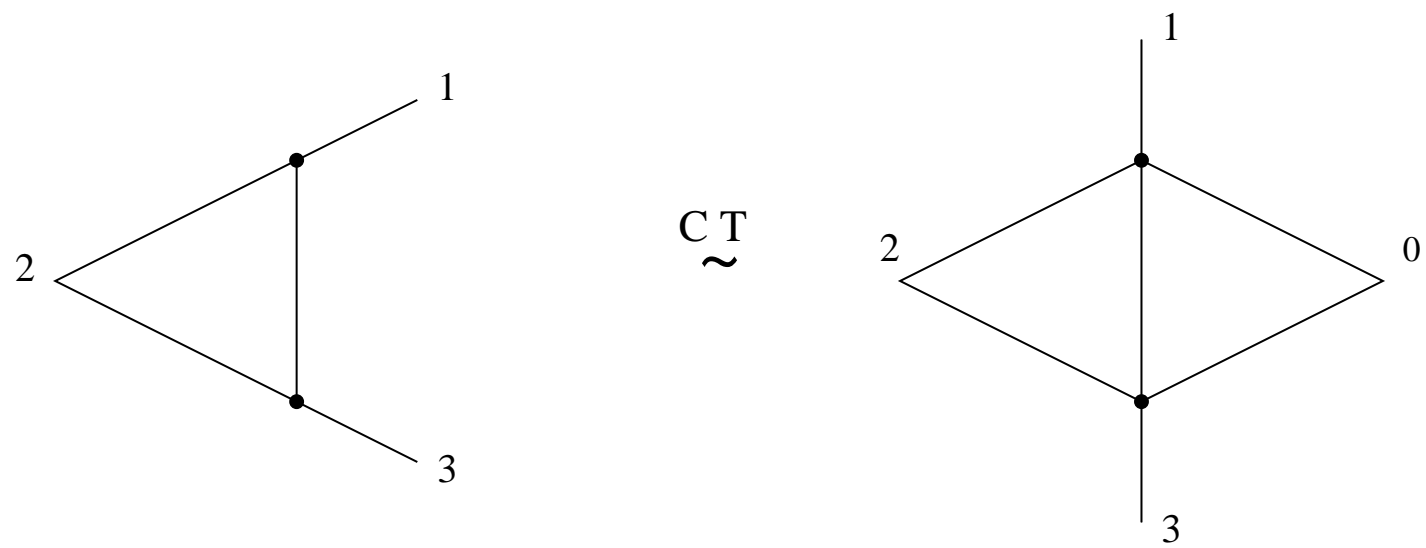

Figure 1: Initial conformal transformation in the position space

according to Eq. (1) is

$$
\begin{array}{r}
\int d^{4} y d^{4} z \frac{1}{[2 y][y z][2 z][3 z][1 y]}= \\
{\left[2^{\prime}\right]^{2}\left[3^{\prime}\right]\left[1^{\prime}\right] \int d^{4} y^{\prime} d^{4} z^{\prime} \frac{1}{\left[2^{\prime} y^{\prime}\right]\left[y^{\prime} z^{\prime}\right]\left[2^{\prime} z^{\prime}\right]\left[3^{\prime} z^{\prime}\right]\left[1^{\prime} y^{\prime}\right]\left[y^{\prime}\right]\left[z^{\prime}\right]}=} \\
{\left[2^{\prime}\right]^{2}\left[3^{\prime}\right]\left[1^{\prime}\right] \frac{1}{\left[3^{\prime} 1^{\prime}\right]\left[2^{\prime}\right]^{2}} \Phi^{(2)}\left(\frac{\left[1^{\prime} 2^{\prime}\right]\left[3^{\prime}\right]}{\left[3^{\prime} 1^{\prime}\right]\left[2^{\prime}\right]}, \frac{\left[1^{\prime}\right]\left[2^{\prime} 3^{\prime}\right]}{\left[3^{\prime} 1^{\prime}\right]\left[2^{\prime}\right]}\right)=} \\
\frac{\left[3^{\prime}\right]\left[1^{\prime}\right]}{\left[3^{\prime} 1^{\prime}\right]} \Phi^{(2)}\left(\frac{\left[1^{\prime} 2^{\prime}\right]\left[3^{\prime}\right]}{\left[3^{\prime} 1^{\prime}\right]\left[2^{\prime}\right]}, \frac{\left[1^{\prime}\right]\left[2^{\prime} 3^{\prime}\right]}{\left[3^{\prime} 1^{\prime}\right]\left[2^{\prime}\right]}\right)=\frac{1}{[31]} \Phi^{(2)}\left(\frac{[12]}{[31]}, \frac{[23]}{[31]}\right) .
\end{array}
$$

The second row of this chain of transformations looks like the second UD integral in the dual representation of Ref. [17]. It corresponds to the r.h.s. of Fig. (1). The last line is the conformal transformation back to the initial variables. Thus, we have proved the formula

$$
\int d^{4} y d^{4} z \frac{1}{[2 y][1 y][3 z][y z][2 z]}=\frac{1}{[31]} \Phi^{(2)}\left(\frac{[12]}{[31]}, \frac{[23]}{[31]}\right) .
$$

After making Fourier transformation, we have the following representation for the l.h.s. (definitions of momenta are indicated on Fig. (2)):

$$
\begin{array}{r}
\int d^{4} y d^{4} z \frac{1}{[2 y][1 y][3 z][y z][2 z]}=4 \pi^{2} \int d^{4} p_{1} d^{4} p_{2} d^{4} p_{3} d^{4} r \delta\left(p_{1}-p_{2}+p_{3}\right) \times \\
\times \frac{1}{p_{1}^{2} p_{3}^{2}} e^{i p_{2} x_{2}} e^{-i p_{1} x_{1}} e^{-i p_{3} x_{3}} \frac{1}{\left(r+p_{1}\right)^{2} r^{2}\left(r-p_{3}\right)^{2}}= \\
4 \pi^{2} \int d^{4} p_{1} d^{4} p_{2} d^{4} p_{3} \delta\left(p_{1}-p_{2}+p_{3}\right) e^{i p_{2} x_{2}} e^{-i p_{1} x_{1}} e^{-i p_{3} x_{3}} \\
\times \frac{1}{p_{2}^{2} p_{1}^{2} p_{3}^{2}} \Phi^{(1)}\left(\frac{p_{1}^{2}}{p_{2}^{2}}, \frac{p_{3}^{2}}{p_{2}^{2}}\right) .
\end{array}
$$




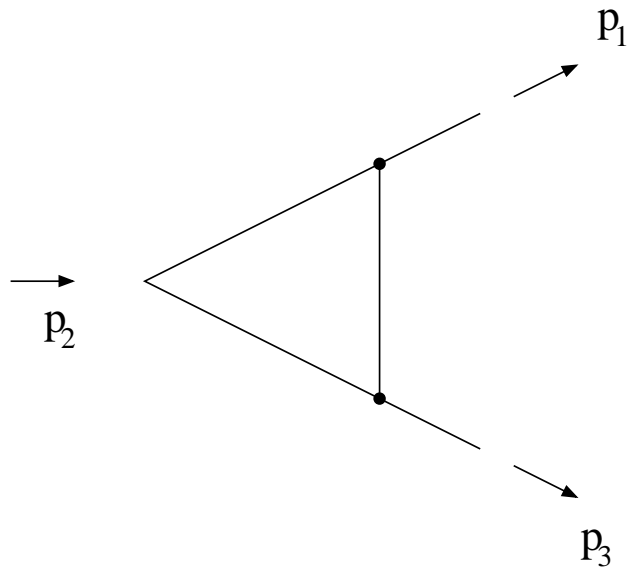

Figure 2: One loop diagram in the momentum space

Thus, comparing with Eq.(2), we can derive the first relation:

$$
\begin{array}{r}
\frac{1}{[31]} \Phi^{(2)}\left(\frac{[12]}{[31]}, \frac{[23]}{[31]}\right)=4 \pi^{2} \int d^{4} p_{1} d^{4} p_{2} d^{4} p_{3} \delta\left(p_{1}-p_{2}+p_{3}\right) \times \\
\times e^{i p_{2} x_{2}} e^{-i p_{1} x_{1}} e^{-i p_{3} x_{3}} \frac{1}{p_{2}^{2} p_{1}^{2} p_{3}^{2}} \Phi^{(1)}\left(\frac{p_{1}^{2}}{p_{2}^{2}}, \frac{p_{3}^{2}}{p_{2}^{2}}\right) .
\end{array}
$$

However, looking at the definition of the UD integrals in Refs. [15, 16], we can write from Eq. (3) another relation:

$$
\begin{aligned}
\frac{1}{[31]^{2}} \Phi^{(2)}\left(\frac{[12]}{[31]}, \frac{[23]}{[31]}\right)= & \frac{1}{16 \pi^{4}} \int d^{4} p_{1} d^{4} p_{2} d^{4} p_{3} \delta\left(p_{1}-p_{2}+p_{3}\right) \times \\
& \times e^{i p_{2} x_{2}} e^{-i p_{1} x_{1}} e^{-i p_{3} x_{3}} \frac{1}{\left(p_{2}^{2}\right)^{2}} \Phi^{(2)}\left(\frac{p_{1}^{2}}{p_{2}^{2}}, \frac{p_{3}^{2}}{p_{2}^{2}}\right) .
\end{aligned}
$$

The next two sections demonstrate how to derive the formula (2) by other two different methods.

\section{Graphical identity}

First of all, we show validity of the graphical identity of Fig.(3). This is identity in the position space. We assume integration over internal vertices. This identity can be proved in two ways. 1. First way to prove Fig.(3) is to use a relation on Fig.(4). This is a graphical representation of the equation (rules of the integration are taken from Ref. [5])

$$
\partial_{(y)}^{2} \frac{1}{[1 y]^{1-\epsilon}}=k(\epsilon) \delta^{(4-2 \epsilon)}(1 y)
$$

from Ref [7]. The coefficient between the 1.h.s. and the r.h.s. of Fig. (4) is $k=-4$ in the number of dimensions $d=4$. On the other side, d'Alambertian can travel along the propagator. 

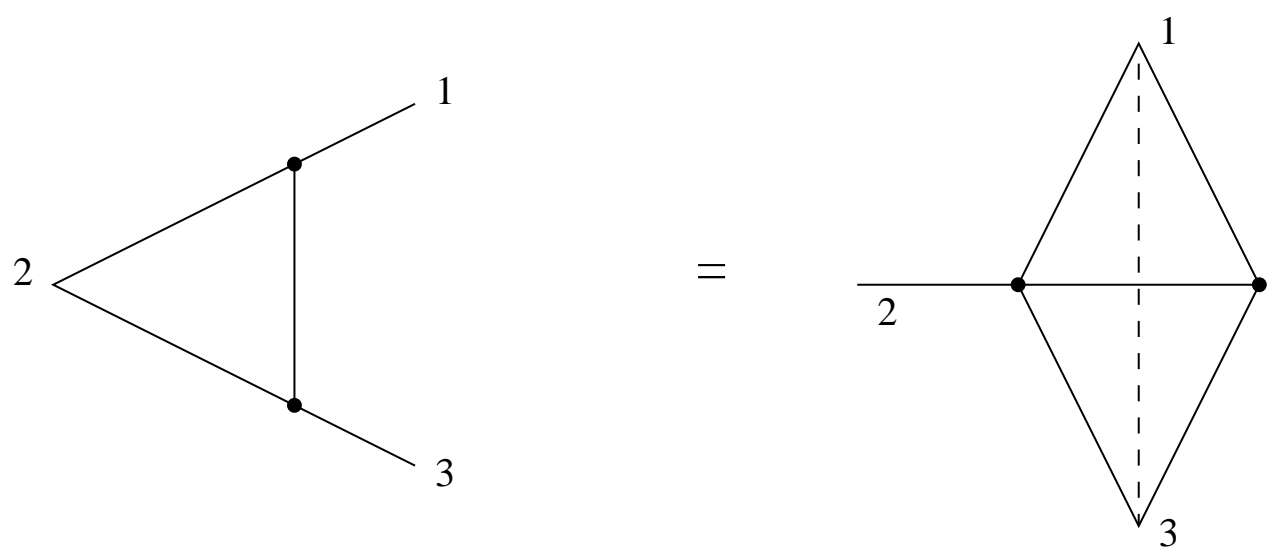

Figure 3: Graphical identity

Computer program of Ref. [7], written in Mathematica, produces the equation ${ }^{2}$

$$
\partial_{(2)}^{2} \int D y D z \frac{1}{[2 y][1 y][3 z][y z][2 z]}=-\frac{4[31]}{[12][23]} J(1,1,1) .
$$

This identity is depicted on Fig.(5). The dash lines correspond to the inversed propagator. The
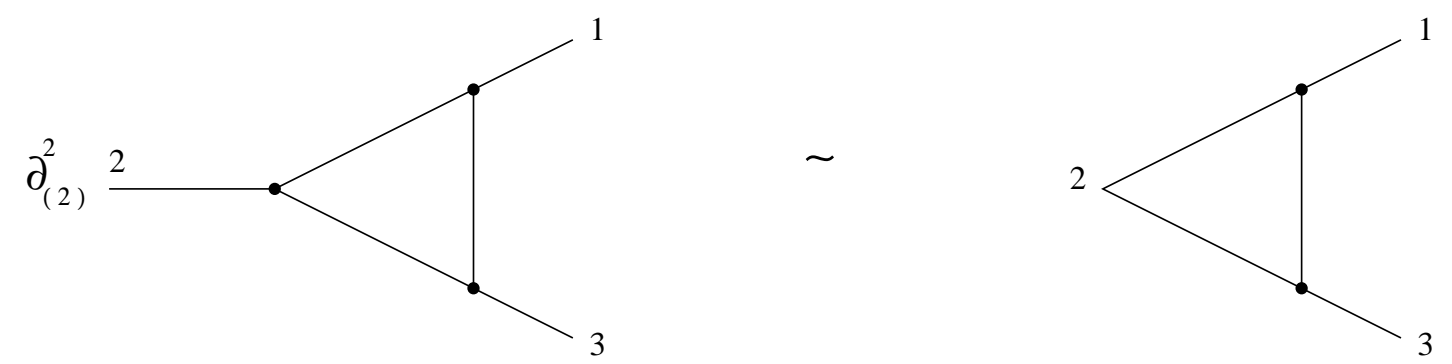

Figure 4: Use of d'Alambertian

coefficient between the 1.h.s. and the r.h.s. of Fig.(5) is $k=-4$. Combining Fig.(4) and Fig.(5) we reproduce the graphical identity of Fig.(3). Integration in the internal vertices includes powers of $\pi$ due to the definition of Ref. [5]. Since both parts of Fig.(3) contain two internal vertices, the identity of Fig.(3) is valid for a usual four-dimensional measure of integration. Thus, we have proved the formula:

$$
\int d^{4} y d^{4} z \frac{1}{[2 y][1 y][3 z][y z][2 z]}=[31] \int d^{4} y d^{4} z \frac{1}{[2 y][y z][1 y][3 y][1 z][3 z]} .
$$

This formula corresponds to the graphical identity of Fig.(3).

2. Another way to show validity of Fig.(3) is a useful identity of Ref. [17] in the position space which can be obtained from the property that $\Phi^{(2)}$ function depends on two conformally

\footnotetext{
${ }^{2}$ The 1.h.s. of (5) appears on page 24 of Ref.[7]
} 


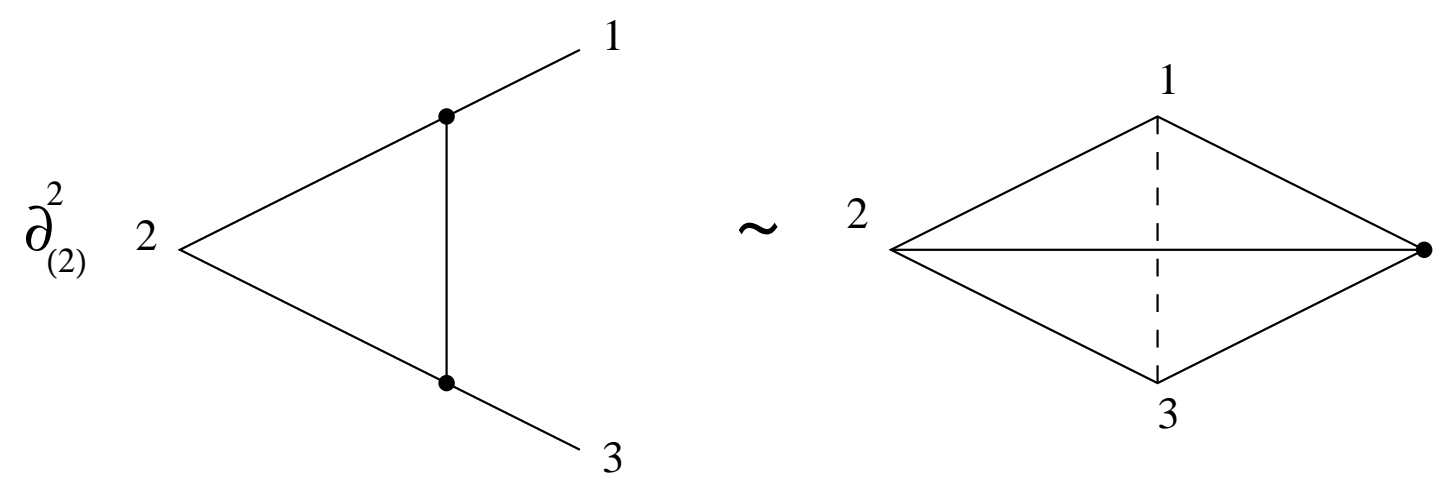

Figure 5: Use of d'Alambertian

invariant ratios of spacetime intervals. This representation is valid in the position space. The turning identity is re-presented in Fig. (6). Historically it appeared in Ref.[17] as a "dual" representation of the momentum two-loop UD integral which is not exactly the same as a position representation (the position representation is usual Feynman ladder diagram integrated over coordinates of internal vertices). Internal vertices correspond to the momenta that run into the loops. However, in the dual representation the integrations are done over "coordinates" of the internal vertices too and thus the dual diagram can be considered as another Feynman diagram in the position space. By multiplying both parts of the turning identity by propagator

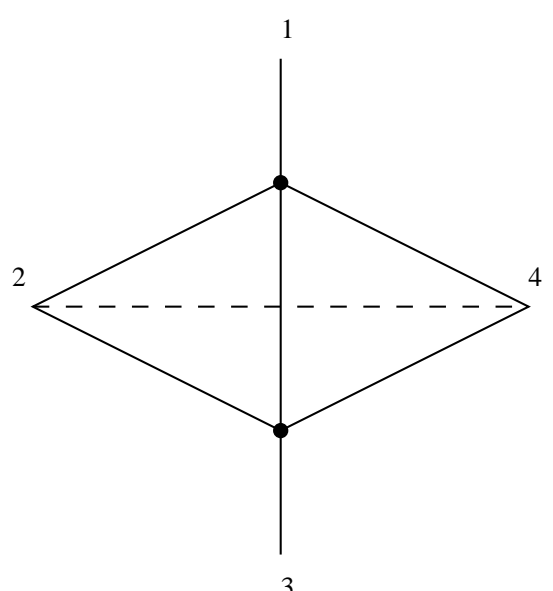

3

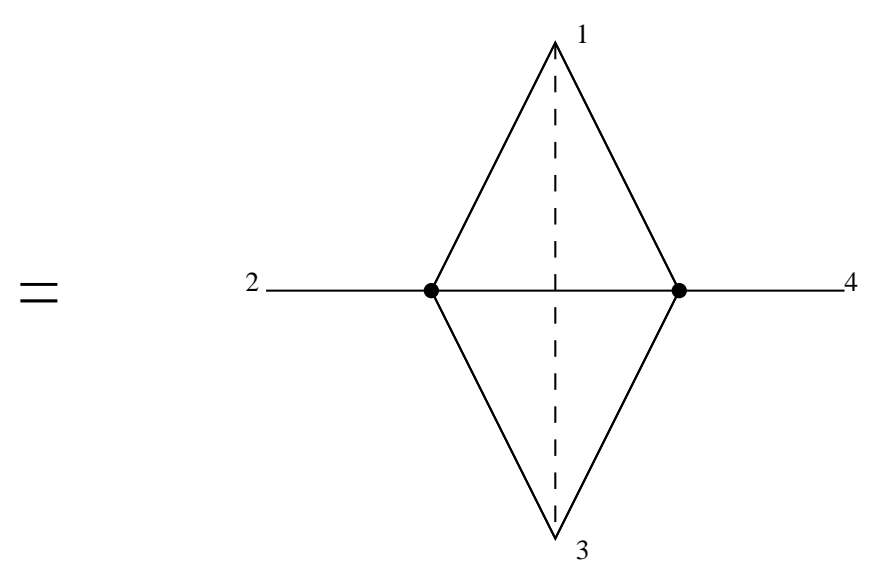

Figure 6: Turning identity.

[24], we have another graphical identity depicted on Fig. (7). Integrating both parts over the variable $x_{4}$ in $d=4-2 \epsilon$ dimensions, we obtain the relation re-presented on Fig. 8, where

$$
A(1,1,2-2 \epsilon)=\frac{\Gamma^{2}(1-\epsilon) \Gamma(\epsilon)}{\Gamma(2-2 \epsilon)}
$$

Cancelling the coefficient $A(1,1,2-2 \epsilon)$ in the both parts and taking the limit $\epsilon \rightarrow 0$, we reproduce Fig.(3). 

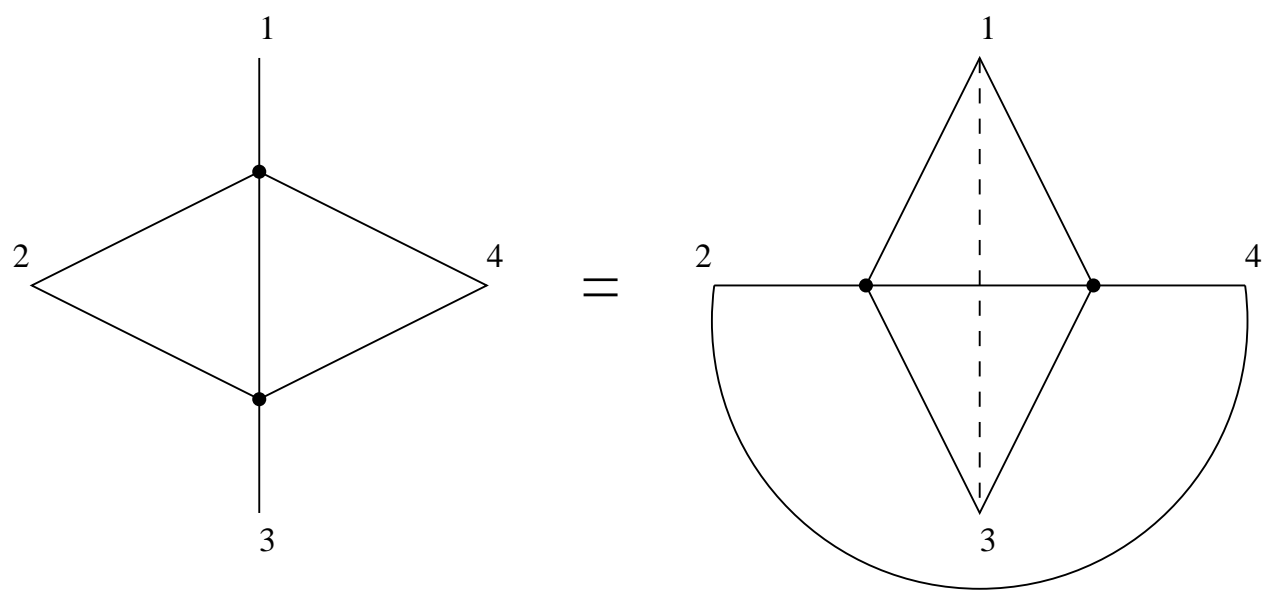

Figure 7: Multiplication by propagator.

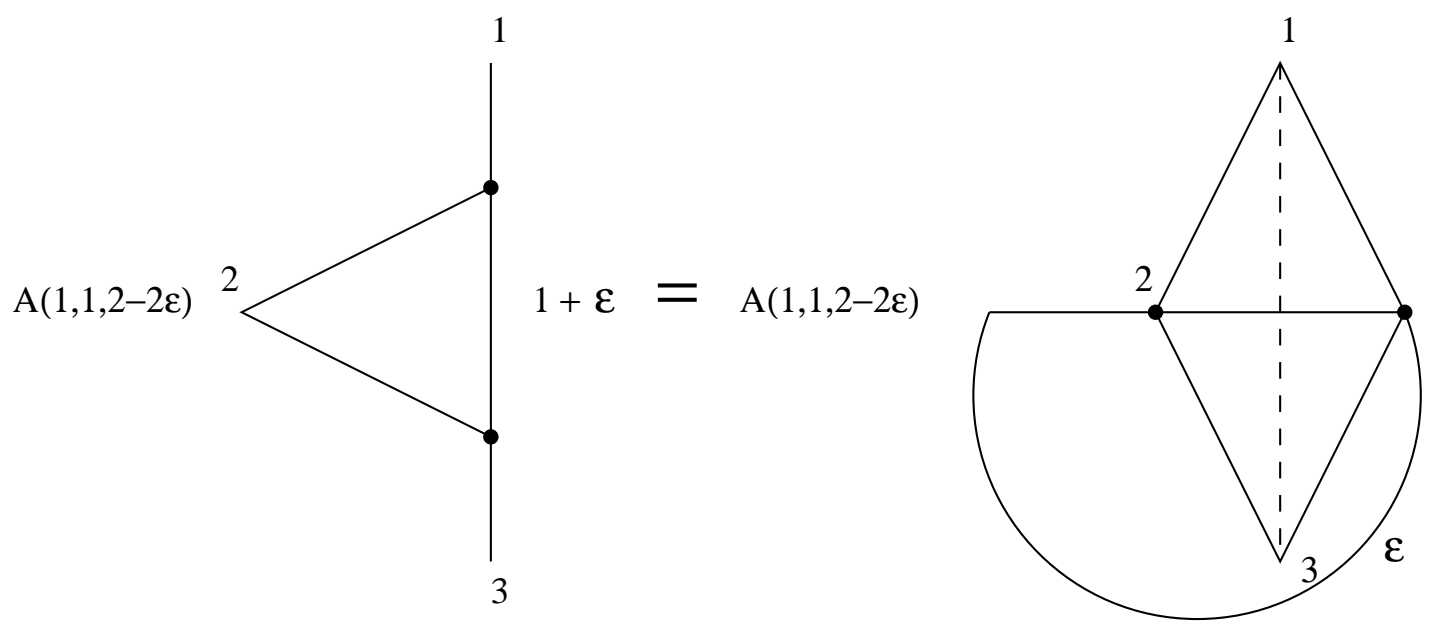

Figure 8: Integration over $x_{4}$.

\section{Relation between graphical identity and UD integral}

After proving Fig.(3), we can relate its r.h.s. to UD integrals. This can be done in two ways. 1. First way is by conformal transformation of the r.h.s. of Fig. (3). Indeed, the integral that corresponds to the r.h.s. of Fig.(3) is

$$
\int d^{4} y d^{4} z \frac{1}{[2 y][y z][1 y][3 y][1 z][3 z]} .
$$

According to the conformal substitution of Eq.(1) the integral of the r.h.s. of Fig. (3) can be transformed to

$$
\int d^{4} y d^{4} z \frac{1}{[2 y][y z][1 y][3 y][1 z][3 z]}=
$$




$$
\begin{gathered}
\int \frac{d^{4} y^{\prime} d^{4} z^{\prime}}{\left[y^{\prime}\right]^{4}\left[z^{\prime}\right]^{4}} \frac{\left[2^{\prime}\right]\left[3^{\prime}\right]^{2}\left[1^{\prime}\right]^{2}\left[y^{\prime}\right]^{4}\left[z^{\prime}\right]^{3}}{\left[2^{\prime} y^{\prime}\right]\left[y^{\prime} z^{\prime}\right]\left[1^{\prime} y^{\prime}\right]\left[3^{\prime} y^{\prime}\right]\left[1^{\prime} z^{\prime}\right]\left[3^{\prime} z^{\prime}\right]}= \\
\int d^{4} y^{\prime} d^{4} z^{\prime} \frac{\left[2^{\prime}\right]\left[3^{\prime}\right]^{2}\left[1^{\prime}\right]^{2}}{\left[2^{\prime} y^{\prime}\right]\left[y^{\prime} z^{\prime}\right]\left[1^{\prime} y^{\prime}\right]\left[3^{\prime} y^{\prime}\right]\left[1^{\prime} z^{\prime}\right]\left[3^{\prime} z^{\prime}\right]\left[z^{\prime}\right]}
\end{gathered}
$$

The transformation is presented on Fig. (9). The r.h.s. of Fig. (9) looks like the second UD integral in the dual representation of Ref. [17]. Thus, we can represent Eq. (7) as
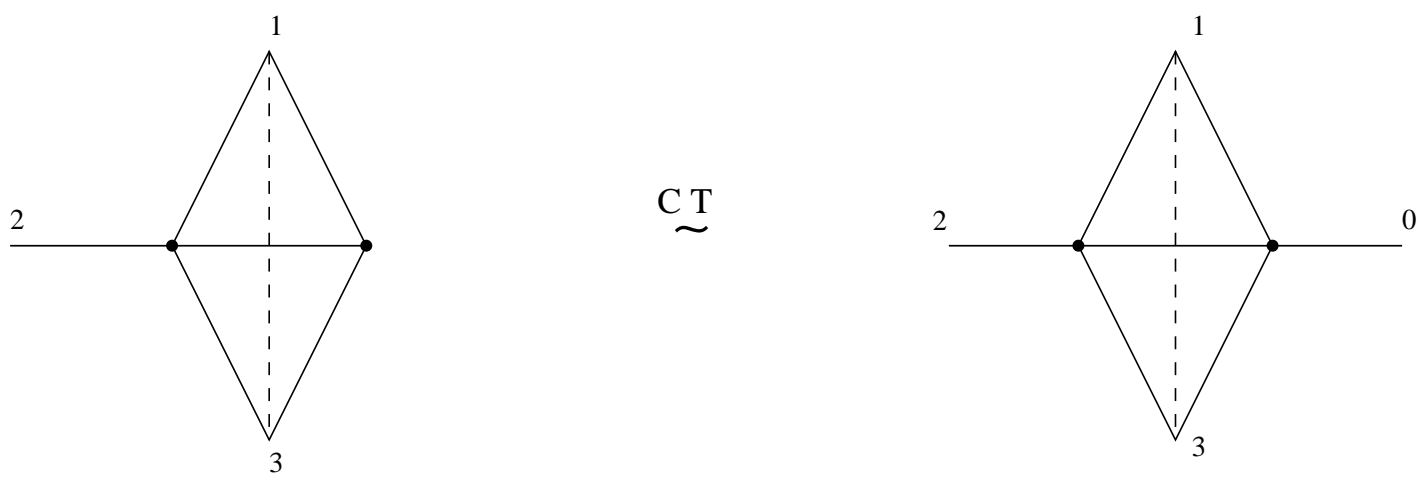

Figure 9: Conformal transformation of the r.h.s. of Fig. 3

$$
\begin{array}{r}
\int d^{4} y^{\prime} d^{4} z^{\prime} \frac{\left[2^{\prime}\right]\left[3^{\prime}\right]^{2}\left[1^{\prime}\right]^{2}}{\left[2^{\prime} y^{\prime}\right]\left[y^{\prime} z^{\prime}\right]\left[1^{\prime} y^{\prime}\right]\left[3^{\prime} y^{\prime}\right]\left[1^{\prime} z^{\prime}\right]\left[3^{\prime} z^{\prime}\right]\left[z^{\prime}\right]}= \\
{\left[2^{\prime}\right]\left[3^{\prime}\right]^{2}\left[1^{\prime}\right]^{2} \frac{1}{\left[3^{\prime} 1^{\prime}\right]^{2}\left[2^{\prime}\right]} \Phi^{(2)}\left(\frac{\left[1^{\prime} 2^{\prime}\right]\left[3^{\prime}\right]}{\left[3^{\prime} 1^{\prime}\right]\left[2^{\prime}\right]}, \frac{\left[1^{\prime}\right]\left[2^{\prime} 3^{\prime}\right]}{\left[3^{\prime} 1^{\prime}\right]\left[2^{\prime}\right]}\right)=} \\
\frac{\left[3^{\prime}\right]^{2}\left[1^{\prime}\right]^{2}}{\left[3^{\prime} 1^{\prime}\right]^{2}} \Phi^{(2)}\left(\frac{\left[1^{\prime} 2^{\prime}\right]\left[3^{\prime}\right]}{\left[3^{\prime} 1^{\prime}\right]\left[2^{\prime}\right]}, \frac{\left[1^{\prime}\right]\left[2^{\prime} 3^{\prime}\right]}{\left[3^{\prime} 1^{\prime}\right]\left[2^{\prime}\right]}\right)=\frac{1}{[31]^{2}} \Phi^{(2)}\left(\frac{[12]}{[31]}, \frac{[23]}{[31]}\right) .
\end{array}
$$

The last line is the conformal transformation back to the initial variables of Eq. (1). Thus, we have demonstrated that

$$
\int d^{4} y d^{4} z \frac{1}{[2 y][y z][1 y][3 y][1 z][3 z]}=\frac{1}{[31]^{2}} \Phi^{(2)}\left(\frac{[12]}{[31]}, \frac{[23]}{[31]}\right)
$$

Taking into account Eq.(6), we have proved the formula of Eq. (2)

$$
\int d^{4} y d^{4} z \frac{1}{[2 y][1 y][3 z][y z][2 z]}=\frac{1}{[31]} \Phi^{(2)}\left(\frac{[12]}{[31]}, \frac{[23]}{[31]}\right) .
$$

2. Second way to demonstrate validity of Eq. (6) does not require the conformal transformation. The dual representation for the two-loop diagram on Fig. (10) in the momentum space is given on Fig. (11). Thus, for the case $\alpha=\beta=0$ we have the structure of the dual diagram depicted on Fig. (12). However, using the definition of UD functions of Refs. [15, 16] and taking into account the relation for dual momenta, one can see that the r.h.s. of Fig.(12) is

$$
\frac{1}{[31]^{2}} \Phi^{(2)}\left(\frac{[12]}{[31]}, \frac{[23]}{[31]}\right) \text {. }
$$




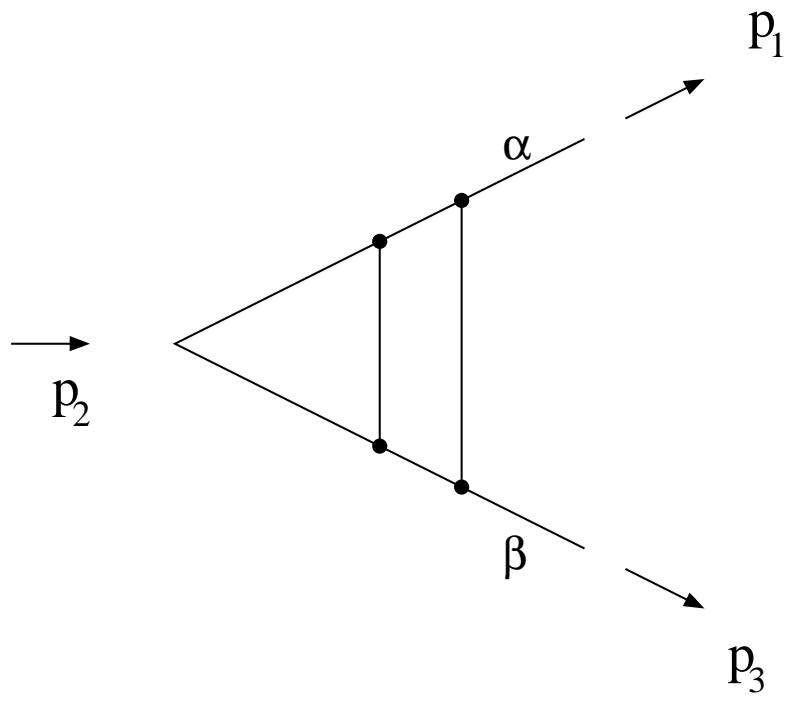

Figure 10: Two-loop diagram

Thus, comparing the l.h.s. of Fig. (12) with the graphical identity Fig.(3) and taking into account Eq. (8) we have in the position space for the l.h.s. of Fig.(3) the result

$$
\frac{1}{[31]} \Phi^{(2)}\left(\frac{[12]}{[31]}, \frac{[23]}{[31]}\right) \text {. }
$$

This coincides with the result in Eq.(2).

\section{Conclusion}

We have shown that Fourier transform of the second UD integral is the second UD integral, and that Fourier transforms of the first and the second UD functions are related. Apart from pure

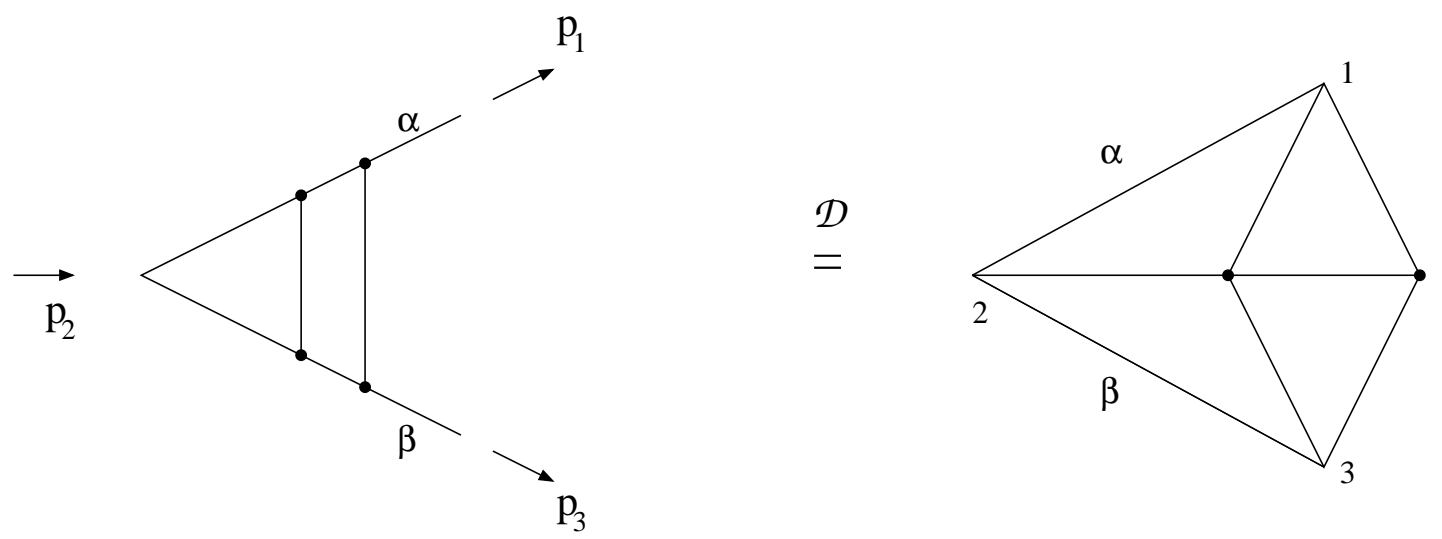

Figure 11: Dual representation 

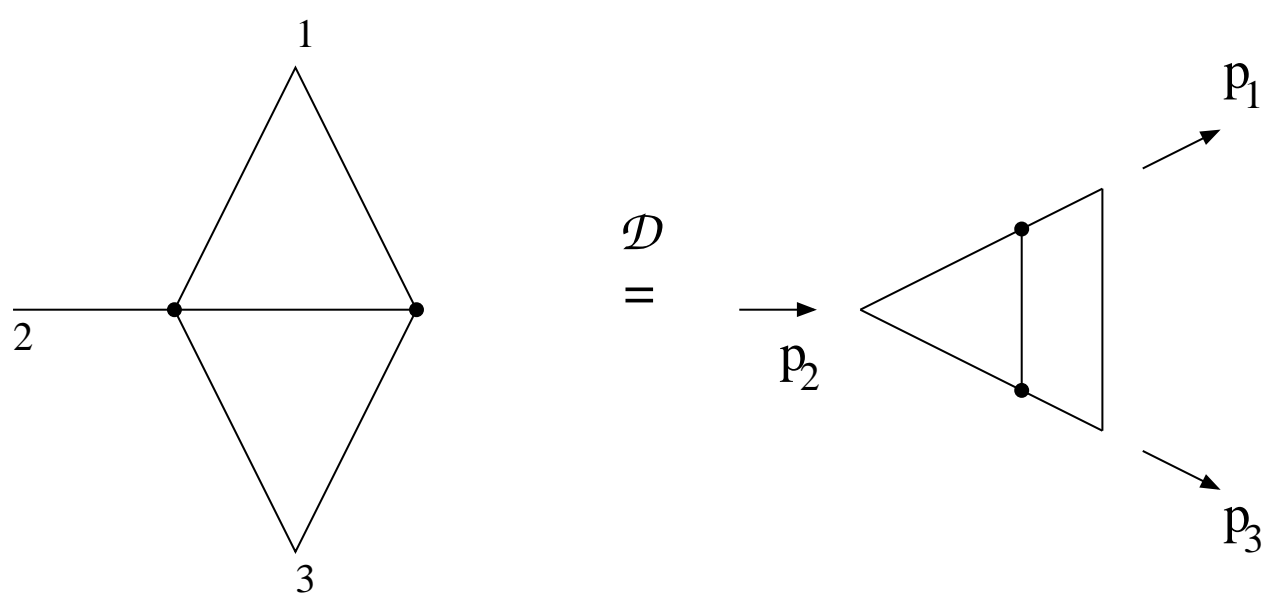

Figure 12: $\alpha=\beta=0$

academic interest, this conclusion allows to investigate correlators of $\mathcal{N}=4$ supersymmetric Yang-Mills theory in the position space. It is useful from the point of view of Slavnov-Taylor identity. This identity will allow to find even maybe yet unknown relations between different types of UD integrals. We hope that the property exists above the present consideration, that is for four-point ladder diagrams and at higher loops too. We plan to consider this in future.

\section{Acknowledgments}

We thank Alvaro Vergara for his work with computer graphics for this paper. I.K. is grateful to organizers of conference "New trends in complex and harmonic analysis" for giving him wonderful opportunity to participate in it and for providing financial support of his stay in Bergen. The work of I.K. was supported by Fondecyt (Chile) project \#1040368, and by Departamento de Ciencias Básicas de la Universidad del Bío-Bío, Chillán (Chile). A.K. is supported by Fondecyt international cooperation project \#7070064.

\section{References}

[1] G. Cvetič, I. Kondrashuk and I. Schmidt, "Effective action of dressed mean fields for N = 4 super-Yang-Mills theory," Mod. Phys. Lett. A 21 (2006) 1127 [hep-th/0407251].

[2] I. Kondrashuk and I. Schmidt, "Finiteness of $\mathrm{n}=4$ super-Yang-Mills effective action in terms of dressed $n=1$ superfields," hep-th/0411150

[3] K. Kang and I. Kondrashuk, "Semiclassical scattering amplitudes of dressed gravitons," hep-ph/0408168.

[4] G. Cvetič, I. Kondrashuk and I. Schmidt, "On the effective action of dressed mean fields for $\mathrm{N}=4$ super-Yang-Mills theory," in Symmetry, Integrability and Geometry: Methods and Applications, SIGMA (2006) 002 [math-ph/0601002]. 
[5] G. Cvetič, I. Kondrashuk, A. Kotikov and I. Schmidt, "Towards the two-loop Lcc vertex in Landau gauge," Int. J. Mod. Phys. A 22 (2007) 1905 [arXiv:hep-th/0604112].

[6] G. Cvetič and I. Kondrashuk, "Further results for the two-loop Lcc vertex in the Landau gauge," J. High Energy Phys. 2 (2008) 023 [arXiv:hep-th/0703138]

[7] G. Cvetic and I. Kondrashuk, "Gluon self-interaction in the position space in Landau gauge," arXiv:0710.5762 [hep-th].

[8] I. Kondrashuk, "An approach to solve Slavnov-Taylor identity in D4 N = 1 supergravity," Mod. Phys. Lett. A 19 (2004) 1291 [gr-qc/0309075].

[9] I. Kondrashuk, G. Cvetič, and I. Schmidt, "Approach to solve Slavnov-Taylor identities in nonsupersymmetric non-Abelian gauge theories," Phys. Rev. D 67 (2003) 065006 [hep$\mathrm{ph} / 0203014]$.

[10] G. Cvetič, I. Kondrashuk and I. Schmidt, "QCD effective action with dressing functions: Consistency checks inperturbative regime," Phys. Rev. D 67 (2003) 065007 [hep$\mathrm{ph} / 0210185]$.

[11] I. Kondrashuk, "The solution to Slavnov-Taylor identities in D4 N = 1 SYM," JHEP 0011, 034 (2000) [hep-th/0007136].

[12] I. Kondrashuk, "Renormalizations in softly broken $N=1$ theories: Slavnov-Taylor identities," J. Phys. A 33 (2000) 6399 [hep-th/0002096].

[13] D. I. Kazakov and A. V. Kotikov, "The method of uniqueness "Multiloop calculation in QCD" Theor. Math. Phys. 73 (1988) 1264 [Teor. Mat. Fiz. 73 (1987) 348];

[14] D. I. Kazakov and A. V. Kotikov, "Total alpha-s correction to deep inelatic scattering crosssection ratio, $\mathrm{R}=$ sigma-L / sigma-t in qcd. Calculation of longitudinal structure function," Nucl. Phys. B 307 (1988) 721 [Erratum-ibid. B 345 (1990) 299].

[15] N. I. Usyukina and A. I. Davydychev, "An Approach to the evaluation of three and four point ladder diagrams," Phys. Lett. B 298 (1993) 363.

[16] N. I. Usyukina and A. I. Davydychev, "Exact results for three and four point ladder diagrams with an arbitrary number of rungs," Phys. Lett. B 305 (1993) 136.

[17] J. M. Drummond, J. Henn, V. A. Smirnov and E. Sokatchev, "Magic identities for conformal four-point integrals," JHEP 0701 (2007) 064 [arXiv:hep-th/0607160].

[18] Z. Bern, L. J. Dixon and V. A. Smirnov, "Iteration of planar amplitudes in maximally supersymmetric Yang-Mills theory at three loops and beyond," Phys. Rev. D 72 (2005) 085001 [hep-th/0505205].

[19] Z. Bern, M. Czakon, L. J. Dixon, D. A. Kosower and V. A. Smirnov, "The Four-Loop Planar Amplitude and Cusp Anomalous Dimension in Maximally Supersymmetric YangMills Theory," Phys. Rev. D 75 (2007) 085010 [arXiv:hep-th/0610248]. 
[20] D. Nguyen, M. Spradlin and A. Volovich, "New Dual Conformally Invariant Off-Shell Integrals," arXiv:0709.4665 [hep-th].

[21] L. F. Alday and J. M. Maldacena, "Gluon scattering amplitudes at strong coupling," JHEP 0706 (2007) 064 [arXiv:0705.0303 [hep-th]]. 